\title{
LIM kinase 1 interacts with myosin-9 and alpha-actinin-4 and promotes colorectal cancer progression
}

\author{
Qing Liao ${ }^{1,2,3}$, Rui Li ${ }^{1,2,3}$, Rui Zhou ${ }^{1,2,3}$, Zhihua Pan ${ }^{2}$, Lijun Xu ${ }^{2}$, Yanqing Ding ${ }^{1,2}$ and Liang Zhao*,1,2 \\ ${ }^{1}$ Department of Pathology, Nanfang Hospital, Southern Medical University, Guangzhou, Guangdong 510515, China and \\ ${ }^{2}$ Department of Pathology, School of Basic Medical Sciences, Southern Medical University, Guangzhou, Guangdong 510515, China
}

Background: LIM kinase 1 (LIMK1) is a key regulator of the cytoskeletal organisation involved in cell proliferation and migration. Even though LIMK1 is frequently dysregulated in epithelial cancers, the role and mechanisms of LIMK1 in colorectal cancer (CRC) remains unclear.

Methods: Immunohistochemical analysis was performed to examine the expression and clinical significance of LIMK1 in CRC samples. Loss- and gain-of-function assay was performed to investigate the effects of aberrant expression on cellular biological behaviour of CRC cells in vitro and in vivo. Immunoblotting and immunoprecipitation was used to screen LIMK1-related signalling pathways and downstream factors.

Results: In this study, our results showed that LIMK1 was upregulated in CRC tissues and localised in both the cytoplasm and the nucleus of CRC cells. Overexpression of LIMK1 in cytoplasmic and nuclear subcellular compartments was closely related to tumour metastasis and poor prognosis of CRC patients. Enhanced expression of cytoplasmic and nuclear LIMK1 significantly increased cell proliferation and migration by driving epithelial-mesenchymal transition and activating the PI3K/Akt signal pathway in vitro as well as promoting growth and metastasis of CRC xenografts, whereas opposite effects were achieved in LIMK1-silenced cells. Furthermore, we identified two tumour metastasis-associated proteins, MYH9 and ACTN4, as direct targets of LIMK1, which were required for a LIMK1-mediated aggressive phenotype.

Conclusions: These findings indicate that LIMK1 plays a critical role in promoting CRC progression at subcellular level. Our findings provide new insights into the metastasis of CRC and advocate for the development of clinical intervention strategies against advanced CRC.

Colorectal cancer (CRC) is one of the most common malignancies worldwide and the third leading cause of cancer death (Siegel et al, 2014). Despite recent advances in the diagnosis and management of CRC, the overall survival of CRC patients has not improved markedly over the past years. Early developed dissemination of micro-metastatic cells is potentially responsible for tumour relapse and metastasis, which is the main cause of mortality and poor prognosis (Gupta and Massague, 2006; Spano et al, 2012). Hence, it is still crucial to uncover the molecular mechanisms underlying CRC progression.

LIM kinase 1 (LIMK1), a member of the LIM kinase protein family, is encoded by a gene located on human chromosomes 7q11.23 (Mizuno et al, 1994). LIMK1 consists of two aminoterminal LIM domains, adjacent PDZ, and proline/serine-rich regions, followed by a carboxyl-terminal kinase domain (Nunoue et al, 1995; Okano et al, 1995). It has been found to phosphorylate

\footnotetext{
*Correspondence: Professor L Zhao; E-mail: liangsmu@foxmail.com

${ }^{3}$ These authors contributed equally to this work.
}

Received 11 March 2017; revised 15 May 2017; accepted 31 May 2017; published online 29 June 2017

(C) 2017 Cancer Research UK. All rights reserved 0007-0920/17 
and consequently deactivate cofilin family protein, which results in reorganisation of the actin cytoskeleton (Ohashi et al, 2000). Considering its significance in the actin cytoskeleton, it is perhaps not surprising that LIMK1 plays central roles in numerous biological processes, particularly in the regulation of cell morphology and motility.

Numerous evidences have demonstrated that LIMK1 is abnormally regulated in various types of human cancers and contributes to cancer progression (Chen et al, 2014; Wan et al, 2014; You et al, 2015). Ectopic expression of LIMK1 has been shown to increase tumour growth, angiogenesis, and the metastatic ability of human breast cancer cells (Bagheri-Yarmand et al, 2006) as well as promote the acquisition of an invasive phenotype in benign prostate epithelial cells (Davila et al, 2003). Selective LIMK inhibitors, such as Pyr1, could prevent the growth of both primary tumours and their metastasis in breast cancer, including those that are taxane-resistant (Prunier et al, 2016). However, systemic administration of LIMK inhibitor did not reduce either primary tumour growth or spontaneous metastasis. Unexpectedly, metastasis of breast cancer cells to the liver was increased after administration of the inhibitor (Li et al, 2013). Therefore, the precise function of LIMK1 in cancer deserves further investigation.

So far, the expression and function of LIMK1 in CRC development and progression has not been well characterised. In this study, we examined the expression and clinical significance of LIMK1 in CRC samples, and then performed loss- and gain-offunction assay to investigate the effects of aberrant expression on cellular biological behaviour of CRC cells in vitro and in vivo. We also provided evidence of LIMK1 regulation of signalling pathways and interaction with cytoskeletal proteins.

\section{MATERIALS AND METHODS}

Cell line preparation. Colorectal cancer cell lines SW480, SW620, HCT116, LS174T, HT29, and LOVO were obtained from the Cell Bank of the Chinese Academy of Sciences (Shanghai, China) and maintained as previously described (Zhao et al, 2010). Additionally, a human CRC cell subline with unique liver metastatic potential, designated as SW480/M5, was established in our laboratory (Zhang et al, 2007). All the cells were cultured in RPMI 1640 (Hyclone, Logan, UT, USA) supplemented with 10\% foetal bovine serum (Gibco-BRL, Invitrogen, Paisley, UK) at $37^{\circ} \mathrm{C}$ with a humidity of $5 \% \mathrm{CO}_{2}$.

Tumour tissue samples. Fresh primary CRC specimens and paired noncancerous colorectal tissue were obtained from the Tumor Tissue Bank of Nanfang Hospital. In each case, a diagnosis of primary CRC had been made, and the patient had undergone elective surgery for CRC in Nanfang Hospital between 2007 and 2010. The study was approved by the Ethics Committee of Southern Medical University and all aspects of the study comply with the Declaration of Helsinki.

Western blot analysis. See Supplementary Materials and Methods for details.

Immunofluorescence. See Supplementary Materials and Methods for details.

Immunohistochemistry. See Supplementary Materials and Methods for details.

Plasmid constructs. See Supplementary Materials and Methods for details.

Stable LIMK1-overexpressing lines. See Supplementary Materials and Methods for detail.
siRNA-mediated gene silencing. See Supplementary Materials and Methods and Supplementary Table S1 for details.

Cell proliferation assays. See Supplementary Materials and Methods for details.

Cell migration analysis. See Supplementary Materials and Methods for details.

Wound healing assay. See Supplementary Materials and Methods for details.

Co-immunoprecipitation. See Supplementary Materials and Methods for details.

Animals, tumour growth assay. See Supplementary Materials and Methods for details.

Lung colonisation assay. See Supplementary Materials and Methods for details.

Statistical analysis. See Supplementary Materials and Methods for details.

\section{RESULTS}

LIMK1 is overexpressed in CRC tissues. LIMK1 expression in eight CRC tissues and paired normal colorectal mucosa was detected by western blot. As shown in Figure 1A, an increase in LIMK1 expression was frequently observed in CRC tissues compared with adjacent non-tumour tissues $(P=0.0151)$. Subcellular localisation of LIMK1 protein by immunofluorescence (IF) revealed obviously nuclear signals in CRC tissues compared with control samples (Figure 1B). We further detected the localisation and expression of LIMK1 in a larger cohort with 78 and 152 paraffin-embedded, archived normal colorectal mucosa and CRC tissues, respectively. LIMK1 overexpression was frequently detected in the cytoplasm and nucleus of malignant epithelial cells (Figure 1C). The positivity rate of cytoplasmic LIMK1 expression, but not nuclear LIMK1, was significantly higher in CRC tissues, compared to that of adjacent non-tumour samples $(P<0.001$; Supplementary Table S2). According to reclassification as described above, higher expression of cytoplasmic LIMK1 was detected in 27 out of $152(17.7 \%)$ CRC cases compared with normal mucosa tissues $(P<0.001$; Supplementary Table S2). Overexpression of nuclear LIMK1 was found in 88 out of 152 (57.9\%) CRC cases compared with 14 out of 78 (17.9\%) in normal mucosa tissues $(P<0.001$; Supplementary Table S2).

Both cytoplasmic and nuclear LIMK1 are associated with progression and poor prognostic phenotype of CRC. To further analyse its correlation with clinical features, the data showed that both cytoplasmic and nuclear overexpression of LIMK1 were closely related to lymph node metastasis ( $\mathrm{N}$ classification), distant metastasis ( $\mathrm{M}$ classification), and tumour recurrence of patients with CRC. In addition, high expression of LIMK1 was also associated with tumour location (Supplementary Table S2). Kaplan-Meier survival analysis revealed that patients with cytoplasmic and nuclear overexpression of LIMK1, but not positive LIMK1, had a worse clinical outcome (Figure 1D; Supplementary Figure S1). Such a relationship was more obvious in patients with higher T stage (i.e., T3 and T4) or negative lymph node status (i.e., N0) (Supplementary Figures S2 and S3). Unfortunately, multivariate analysis did not support LIMK1 overexpression in subcellular compartments as independent prognostic factors for CRC (Supplementary Table S3).

Exogenous LIMK1 localised to the cytoplasm and nucleus contributes to aggressive phenotypes of CRC cells in vitro. We assessed the subcellular localisation and expression of LIMK1 
A

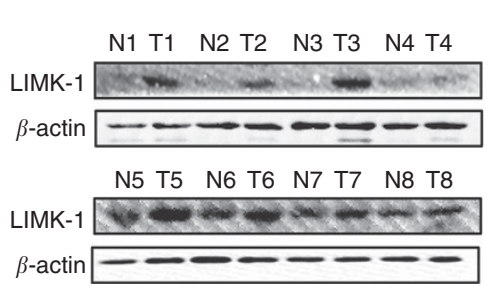

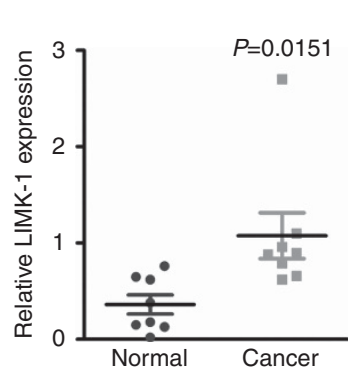

B

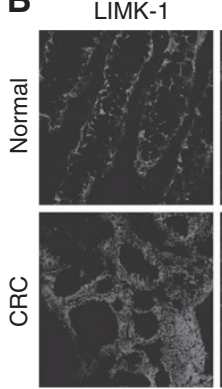

DAPI

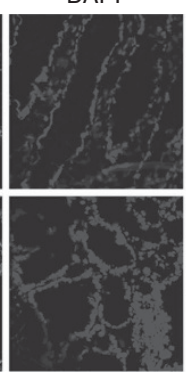

Merge

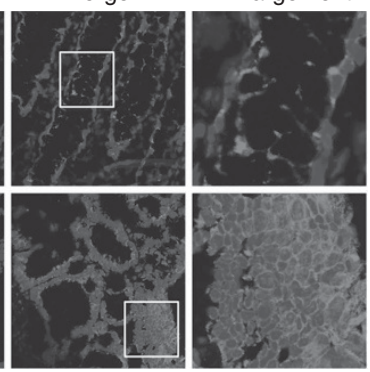

C

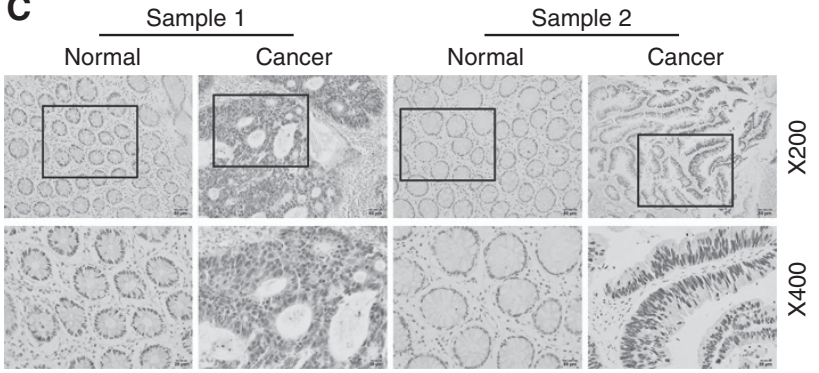

D

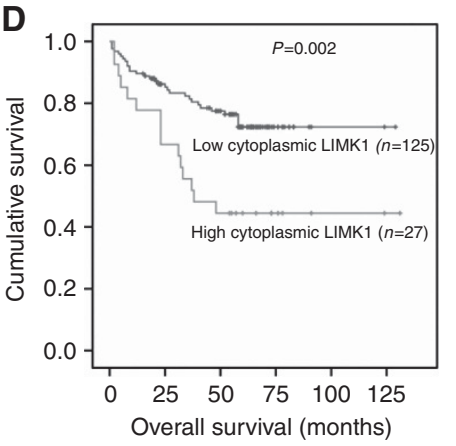

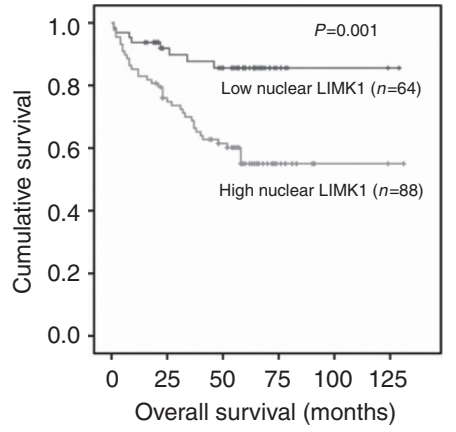

E

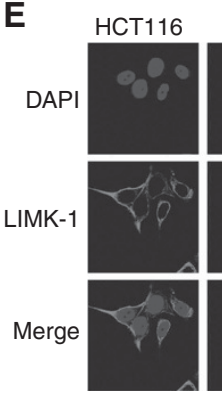

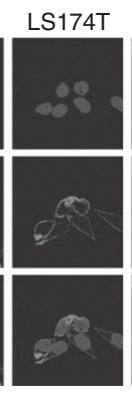
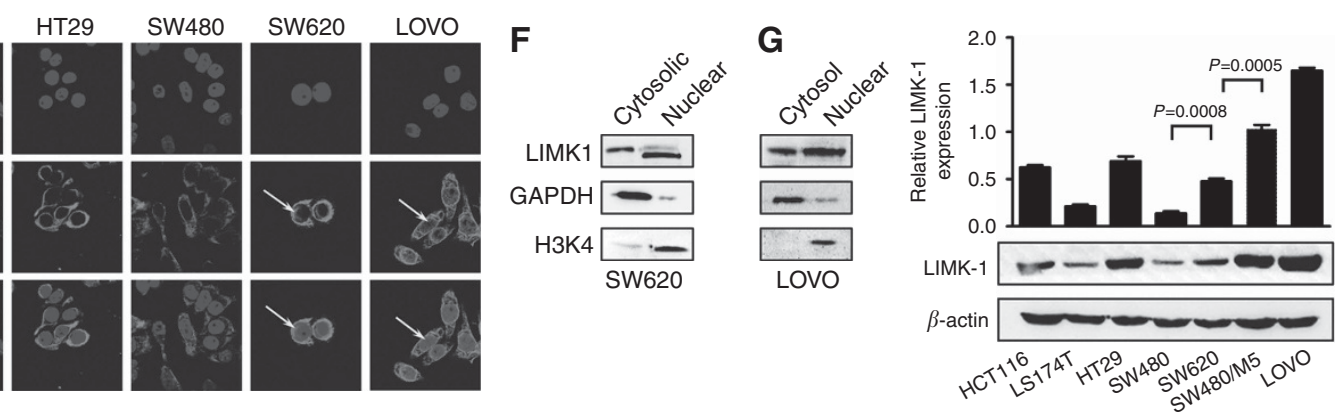

Figure 1. Both cytoplasmic and nuclear LIMK1 are associated with a poor prognostic phenotype of CRCs. (A) Western blot analysis of LIMK1 in CRC tissues ( $T$ ) and adjacent non-tumour tissues ( $N$ ). The immunosignal was quantified using densitometric scanning software, and the relative protein abundance was determined by normalisation with $\beta$-actin. (B) IF analysis of LIMK1 protein expression in normal mucosa tissues and CRC tissues (original magnification $\times 2400$ ). (C) IHC analysis of LIMK1 protein expression in CRC tissues and adjacent non-tumour tissues. Representative photographs of IHC staining in CRC tissues and adjacent non-tumour tissues are shown. (D) Kaplan-Meier survival curves and univariate analyses (log-rank) for CRC patients with distinct expression levels and subcellular localisation of LIMK1. (E) IF analysis for subcellular localisation of LIMK1 in CRC cell lines (original magnification $\times 2400$ ). (F) Western blot analysis of cytoplasmic and nuclear fractions from SW620 and LOVO. Nuclear segregation is assayed by total H3K4. Cytoplasmic segregation is assayed by GAPDH. LIMK1 is assayed with anti-LIMK1 antibody. (G) Western blot analysis for the expression of LIMK1 in CRC cell lines. IHC, immunohistochemistry. A full colour version of this figure is available at the British Journal of Cancer journal online.

protein in all CRC cell lines by IF and western blot assay. Nuclear localisation of LIMK1 was clearly observed in SW620 and LOVO cells with high metastatic potential (indicated as yellow arrow; Figure 1E and F). Compared with HCT116, LS174T, SW480, and HT29 cells with relatively low metastatic potential, LIMK1 expression was also significantly increased in SW620, SW480/M5, and LOVO cells with higher metastatic potential. Interestingly, a gradually increased trend of immunoreactivity was observed in three cell lines, SW480, SW620, and SW480/M5, derived from the same patient, along with an increase in metastatic potential (Figure 1G).

To further investigate the effects of LIMK1 in subcellular compartments on CRC cell behaviours, either a duplicated nuclear export signal (NES) sequence motif or a duplicated nuclear localisation sequence (NLS) was fused in-frame to HA-LIMK1 to generate HA-NES-LIMK1 or HA-NLS-LIMK1, respectively (Figure 2A). In vitro gain-of-function analyses were performed in SW480 and HCT116 cells transiently transfected with recombinant expression vectors. The transfection efficiency and subcellular localisation was confirmed by western blot analysis (Figure 2B; Supplementary Figure S4). The IF assay revealed that the HA-NLSLIMK1 protein was visualised exclusively within the nucleus of SW480 cells. Conversely, the HA-NES-LIMK1 protein was observed by HA visualisation to be excluded from the nucleus. Finally, HALIMK1 was expressed in both the cytoplasm and nucleus (Figure 2C). The CCK-8 assay revealed that exogenous LIMK1, localised to the cytoplasm or/and nucleus, increased the capacity of cell proliferation (Figure 2D). The migration and motility ability was enhanced independently of cytosolic or nuclear expression, as assessed by transwell and wound healing assays (Figure $2 \mathrm{E}$ and F).

In vitro loss-of-function analyses were carried out in SW620 cells with high endogenous expression of LIMK1 using siRNAmediated RNA interference (RNAi). Using western blot analysis, two of the most effective siRNAs (siRNA-391 and siRNA-377) were selected for further examination (Figure $3 \mathrm{~A}$ ). The results of CCK- 8 assays revealed that silencing of LIMK1 could significantly suppress cell proliferation in SW620 cells $(P<0.001$; Figure $3 B)$. 
A

HA HA

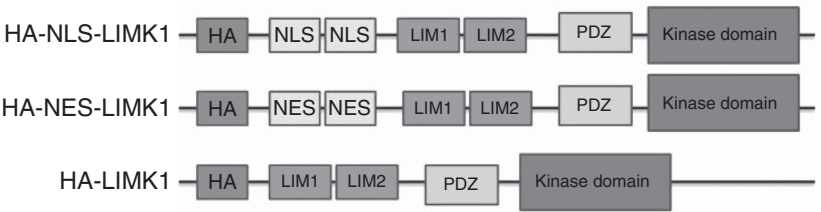

C SW480

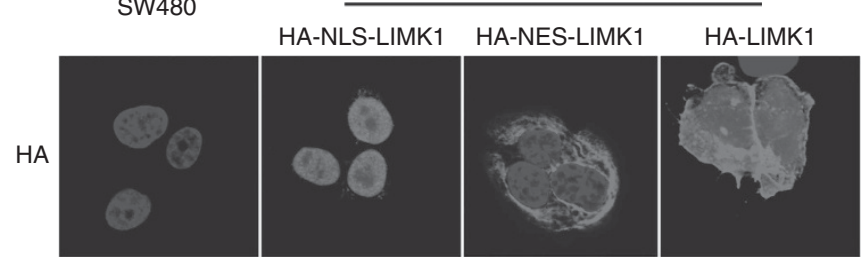

B

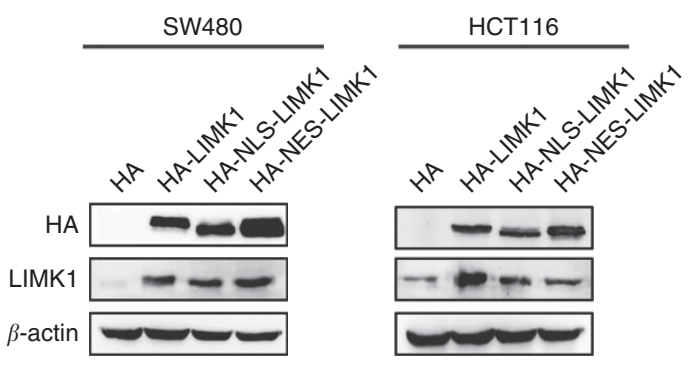

SW480

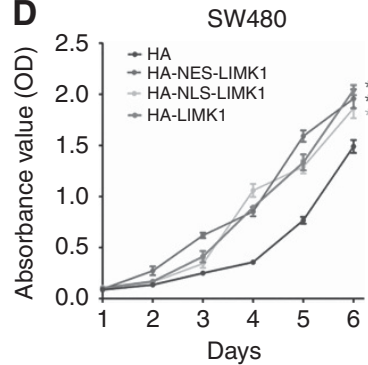

HCT116

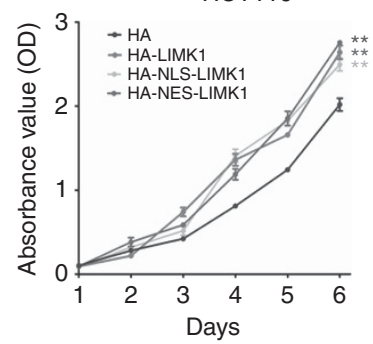

E
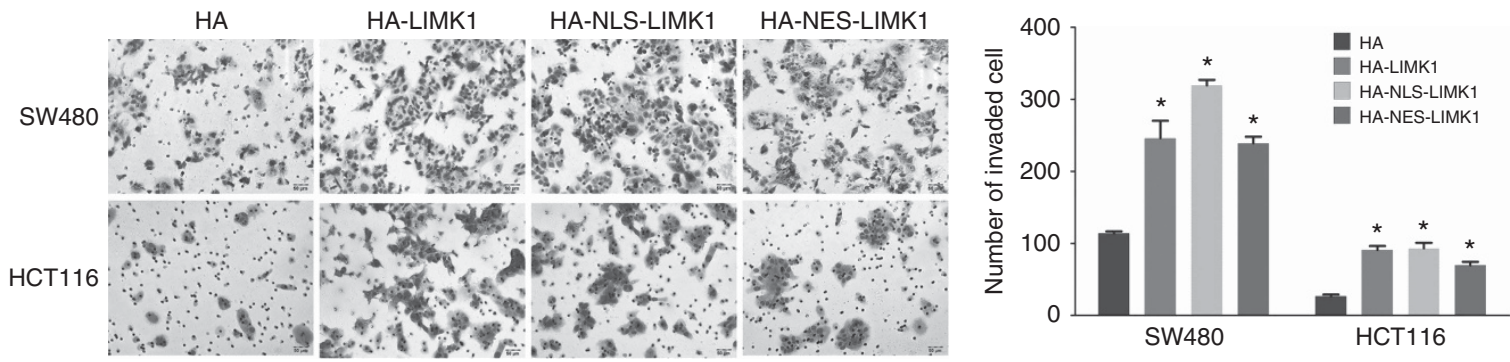

$\mathbf{F}$
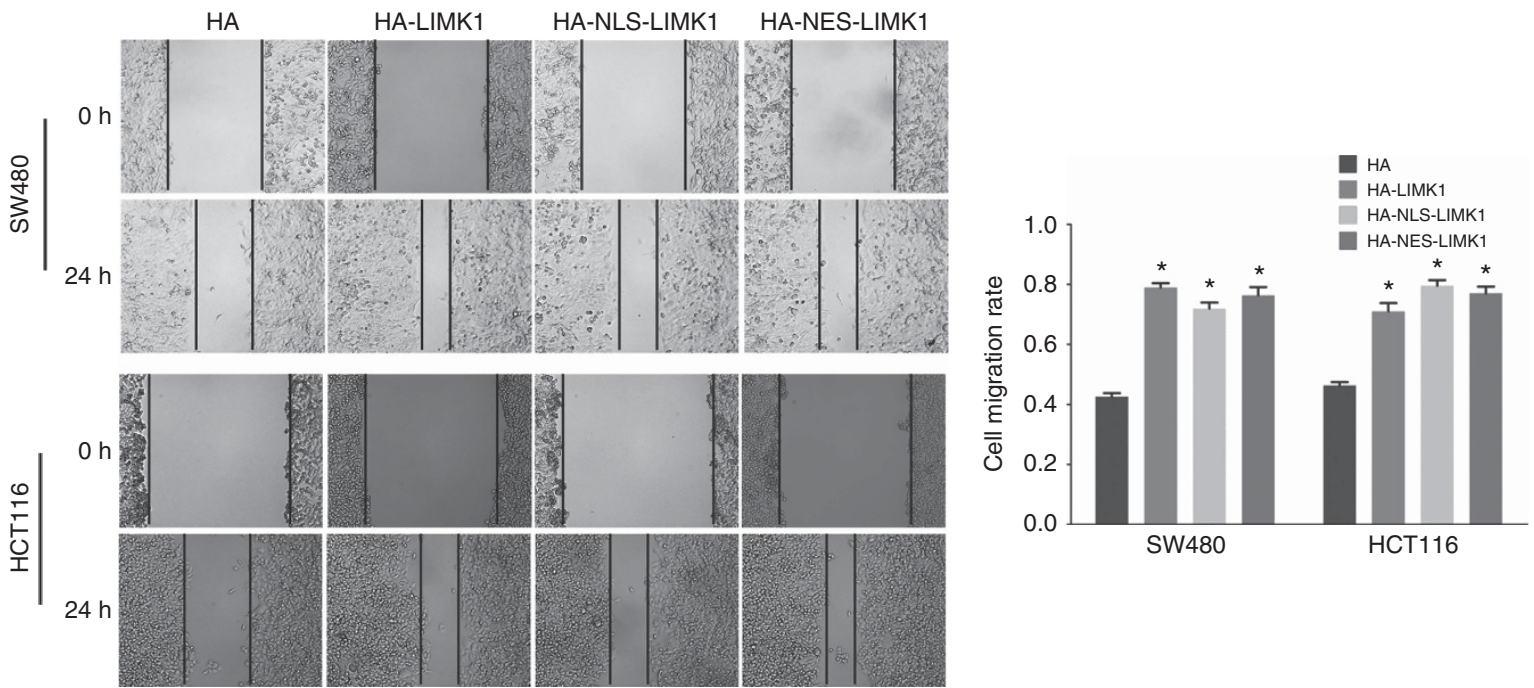

Figure 2. Exogenous LIMK1 localised to the cytoplasm and nucleus contributes to aggressive phenotypes in vitro. (A) HA-alone and three HA-tagged LIMK1 proteins are depicted graphically and colour coded. HA was fused to the N-terminus of LIMK1 cDNA. Exogenous NLS and NES tags were fused to the N-terminus of HA. (B) SW480 and HCT116 cells were transiently transfected with HA, HA-NES-LIMK1, HA-NLS-LIMK1, and HA-LIMK1 vectors. Western blot analysis was performed to detect the exogenous and total expression of LIMK1 using anti-HA and anti-LIMK1. (C) IF assay was used to visualise subcellular localisation of LIMK1 in treated SW480 cells (original magnification $\times 2400$ ). Exogenous NLS and NES sequences target HA-NLS-LIMK1 and HA-NES-LIMK1 proteins to the nuclear and cytoplasmic subcellular compartments. (D) The effect of LIMK1 on cell proliferation was evaluated by CCK-8 assay. (E) The invading cells of the transwell assay were counted under a microscope in five randomly selected fields. Bars represent the number of invaded cells. (F) Wound healing assay was performed to evaluate the motility. Bars represent migration index of treated or control cells. The distance migrated by treated cells was relative to that migrated by control cells. Representative figures are shown. The asterisk $\left(^{*}\right)$ indicates $P<0.05$. The double asterisk $\left(^{\star *}\right)$ indicates $P<0.01$. A full colour version of this figure is available at the British Journal of Cancer journal online. 
A
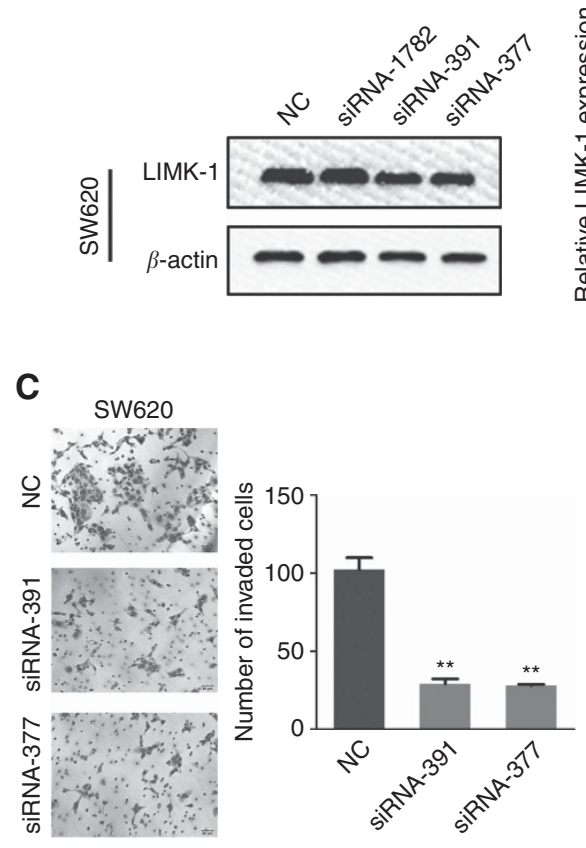
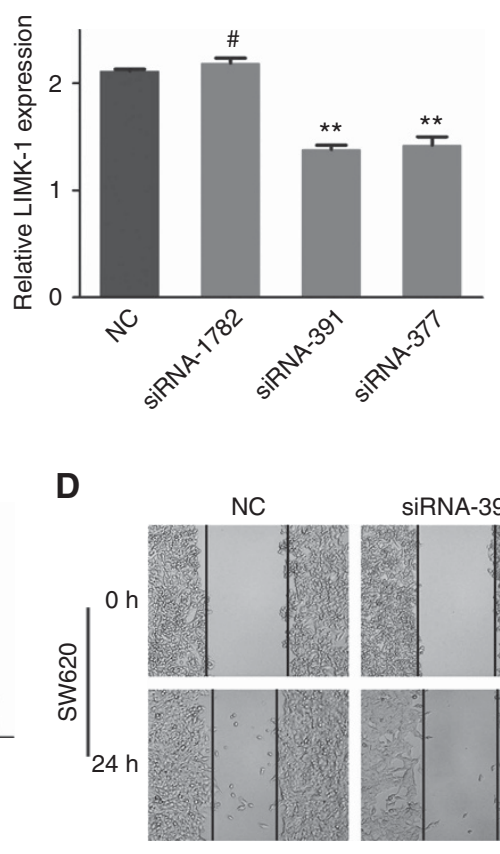

B

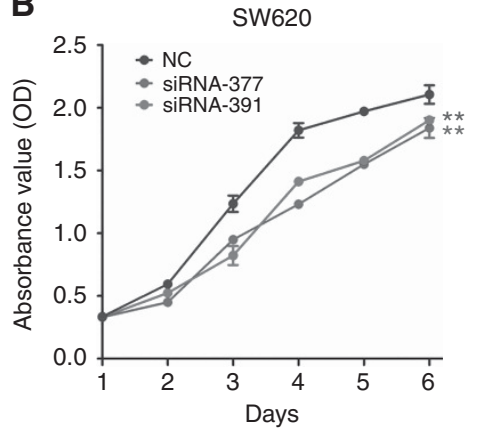

Figure 3. Silencing of LIMK1 suppresses cell proliferation and migration in vitro. (A) SW600 cells were transiently transfected with LIMK1 siRNAs. Western blotting analysis of LIMK1 was performed to evaluate interfering efficiency. (B) The effect of LIMK1 siRNA on cell proliferation was evaluated by CCK-8 assay. (C) The invading cells of the transwell assay were counted under a microscope in five randomly selected fields. Bars represent the number of invaded cells. (D) Wound healing assay was performed to evaluate the motility ability. Bars represent migration index of treated or control cells. The

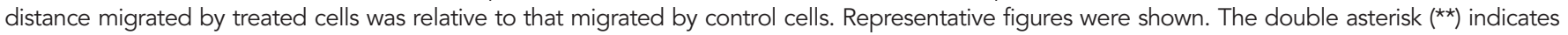
$P<0.01$. The hash (\#) indicates $P>0.05$. A full colour version of this figure is available at the British Journal of Cancer journal online.

The siRNA-treated cells also showed a significant decrease of migration and motility potential in transwell and wound healing assays $(P<0.05$; Figure $3 \mathrm{C}$ and $\mathrm{D})$.

\section{Subcellular localisation of endogenous LIMK1 overexpression promotes CRC growth and progression in vivo. The effect of subcellular localisation of LIMK1 on tumour growth was assessed by subcutaneous injection of tumour cells into the back of nude mice. SW480 CRC cell lines with stable LIMK1 overexpression targeted to the cytoplasm and nucleus were established and successfully validated by western blot and IF assay (Figure 4A and B; Supplementary Figure S5). Expectedly, in vitro experiments confirmed that stably transfectants show an increase in prolif- erative and migratory ability (Supplementary Figure S6). Subse- quently, a subcutaneous tumour model was used to assess the potential of tumour growth. As shown in Figure 4C, the tumours in the SW480/HA-NES-LIMK1, SW480/HA-NLS-LIMK1, and SW480/HA-LIMK1 groups grew obviously faster (Figure 4D), and showed a higher percentage of positive Ki-67 tumour cells compared with those in the SW480/HA group (Figure 4E). \\ The effect of endogenous LIMK1 localised to the cytoplasm and nucleus on the potential of homing capacity was evaluated 60 days after the nude mice had been injected with the same cells through their tail veins. Compared to the control group, more and larger tumour nodules were found in the lungs of mice with LIMK1- overexpressing groups with cytoplasmic or nuclear localisation (Figure 4F).}

LIMK1 localised to the cytoplasm and nucleus induces epithelial-mesenchymal transition and activates the PI3K/Akt signalling pathway. To evaluate the relationship between the expression and activity of LIMK1 in CRC, we have performed western blot analysis to detect the levels of active LIMK1
(phospho-LIMK1), phospho-cofilin, and LIM kinase 2 (LIMK2). As shown in Supplementary Figure S6, accompanied by changes of LIMK1 protein expression, corresponding changes of phosphoLIMK1 and phospho-cofilin, but opposite changes of LIMK2, have occurred (Supplementary Figure S7).

Next, we explored whether LIMK1 introduction has an effect on the expression of markers associated with epithelial-mesenchymal transition (EMT) and the possible oncogenic pathways that may involve the function of LIMK1. Western blot analyses showed that the mesenchymal markers (fibronectin and N-cadherin) and transcription factor snail were upregulated, while epithelial markers (E-cadherin and $\beta$-catenin) were downregulated through introduction of exogenous LIMK1 in cytoplasmic and nuclear compartments (Figure 5A). As shown in Figure 5B, cytoplasmic and nuclear LIMK1 could activate phosphatidylinositol-4,5bisphosphate 3-kinase (PI3K)/Akt signalling via decreased phosphorylation of PTEN and increased phosphorylation of AKT at Ser473 and Thr308 in SW480 and HCT116 cells.

MYH9 and ACTN4 are downstream factors of LIMK1. To identify the unknown downstream molecules of compartmentspecific expression of LIMK1, the whole proteins of whole-cell lysates from SW480 cells stably expressing HA, HA-LIMK1, HANES-LIMK1, and HA-NLS-LIMK1 were extracted and immunoprecipitated with a specific HA-tag antibody. Differential protein bands were revealed by silver staining in the resultant immunoprecipitate and analysed by MS (Figure 6A). The identified protein bands are summarised in Supplementary Table S4. Among them, band \#1 was identified as MYH9 (myosin-9), and band \#2 was identified as ACTN4 (alpha-actinin-4) (Supplementary Figure S8). Both proteins were confirmed by immunoprecipitation and immunoblotting with anti-HA, anti-MYH9, or anti-ACTN4 antibody (Figure 6B). Immunofluorescence assays validated subcellular 


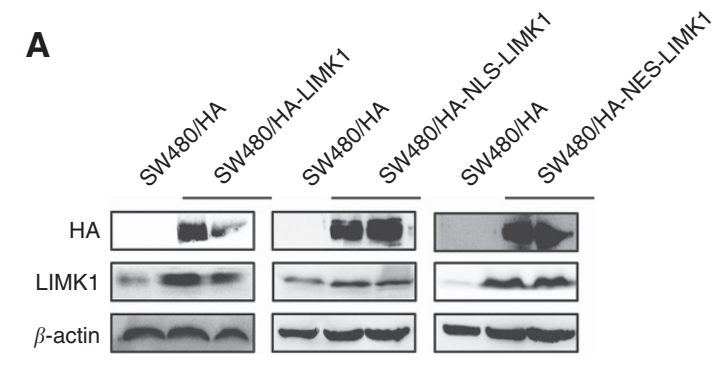

B
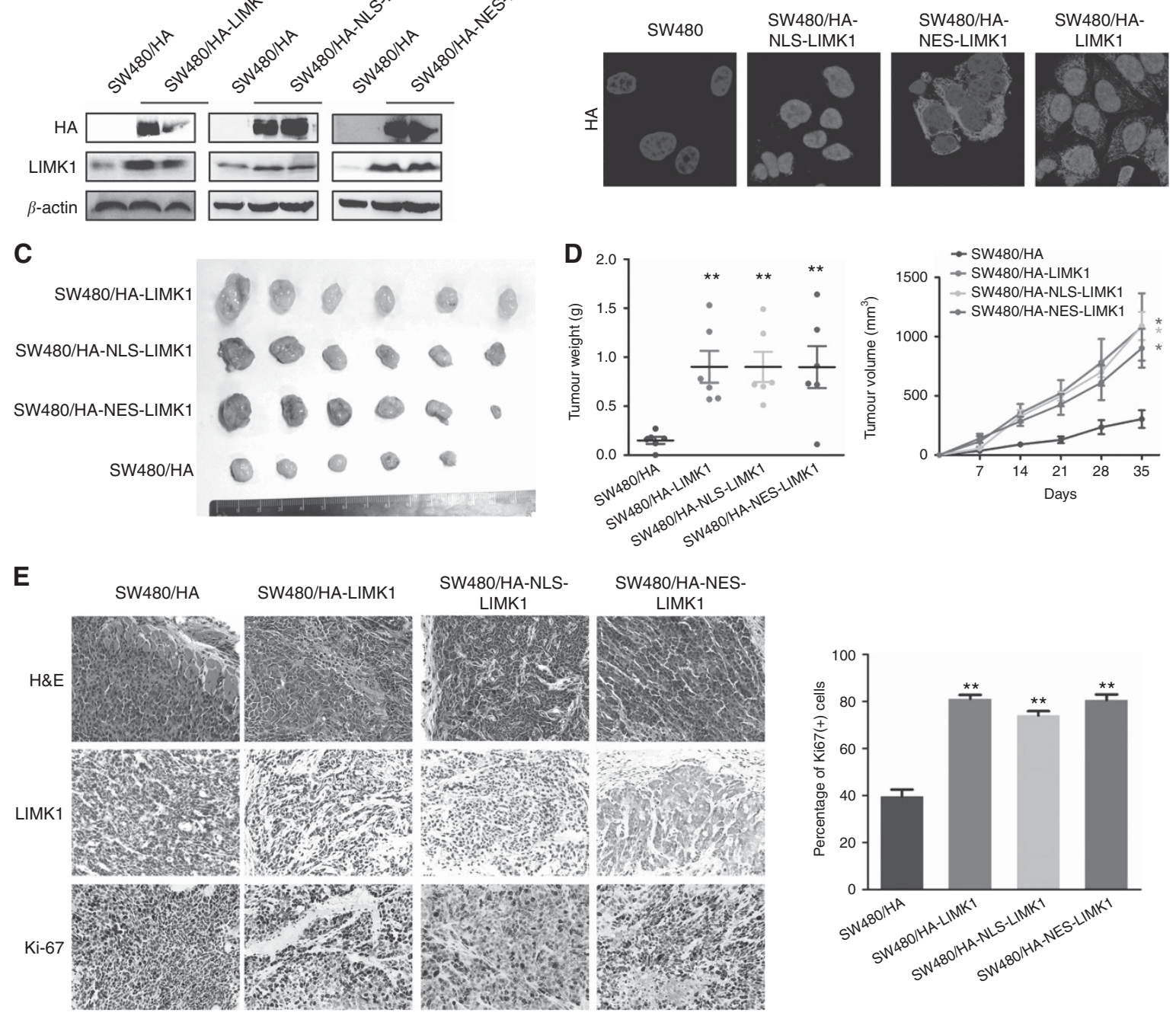

$\mathbf{F}$
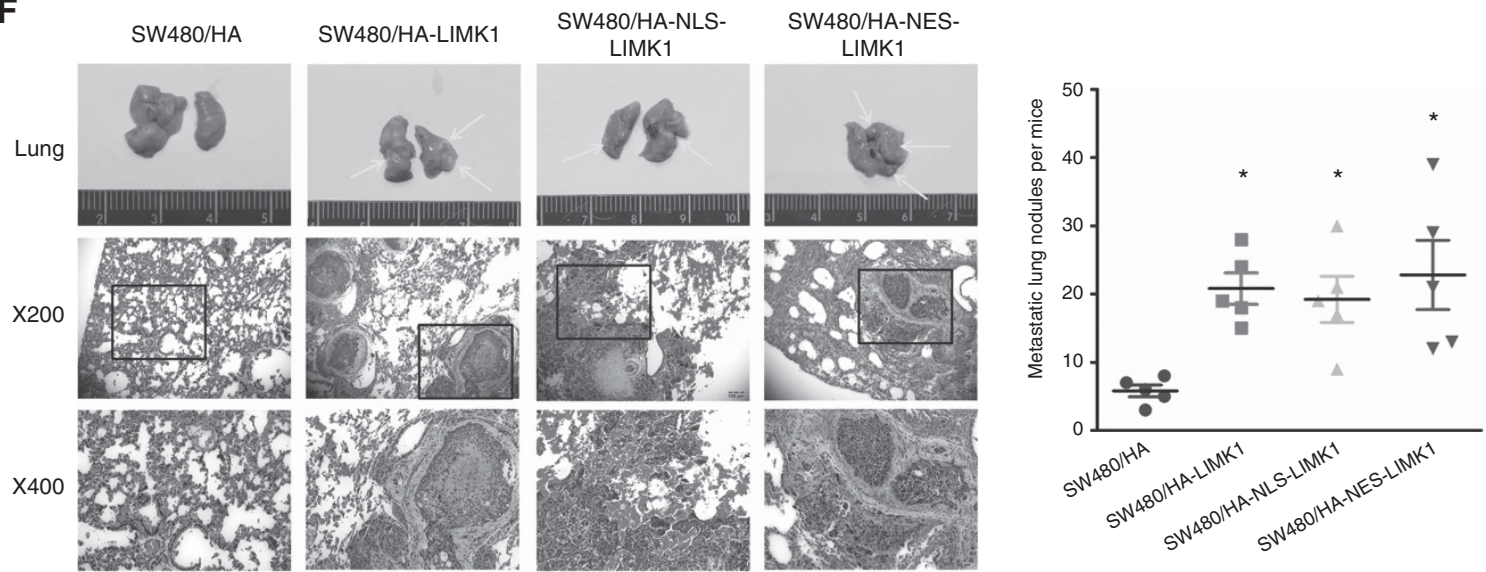

Figure 4. Subcellular localisation of endogenous LIMK1 overexpression promotes CRC growth and progression in vivo. (A) Western blot analysis was used to detect the endogenous overexpression of LIMK1 in stable subcellular transfectants. (B) IF assay was used to visualise subcellular localisation of LIMK1 in stable transfectants (original magnification $\times 2400$ ). (C) Tumour cells were injected subcutaneously into the back of nude mice to evaluate tumour growth. Representative figure of tumours formed is shown. (D) Tumour weight and volume in the back of nude mice injected with indicated cells was measured. The data of all primary tumours are expressed as mean \pm s.d. Scatter plots of tumour weight derived from indicated cells at $30 \mathrm{~d}$ after subcutaneous implantation. (E) The representative photographs of H\&E and LIMK1 staining of subcutaneous tumour are shown. Proliferative ability was measured by the Ki-67 proliferative labelling index. (F) Tumour cells were injected into nude mice through the tail vein to evaluate the lung homing potential of cells. The number of metastatic lung nodules in individual mice was counted under the microscope. The magnification areas indicated metastatic nodes in the lung. The asterisk (*) indicates $P<0.05$. The double asterisk (**) indicates $P<0.01$. A full colour version of this figure is available at the British Journal of Cancer journal online. 

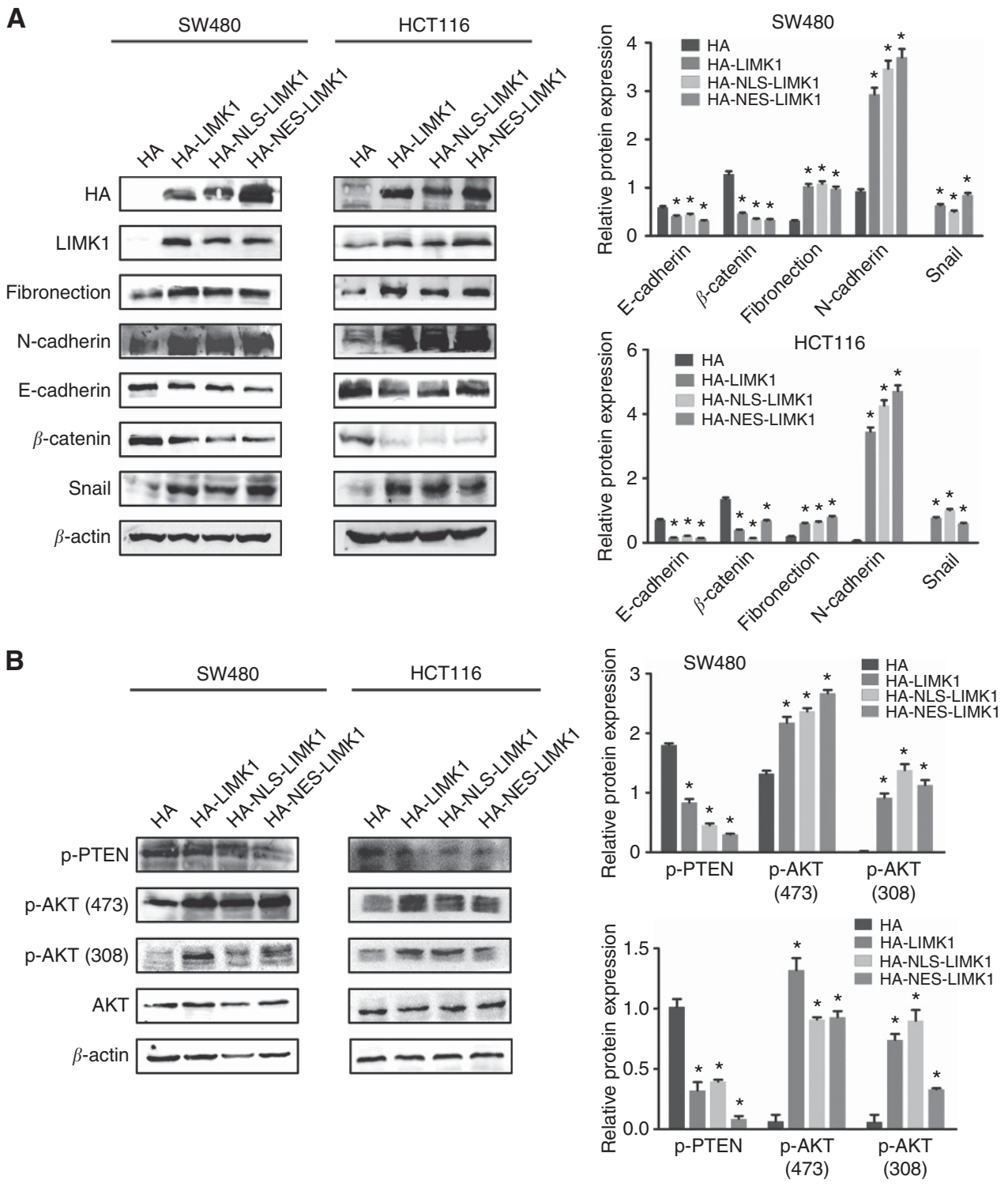

Figure 5. LIMK1 localised to the cytoplasm and nucleus induces EMT and activates the PI3K/Akt signalling pathway. (A) Western blot analysis of EMT markers (E-cadherin, $\beta$-catenin, vimentin, N-cadherin, snail) in indicated cells treated with LIMK1 vector. (B) Western blot analysis of phosphorylated PTEN and AKT at Ser473 and Thr308 in indicated cells treated with LIMK1 vector. The immunosignal was quantified using densitometric scanning software, and relative protein abundance was determined by normalisation with $\beta$-actin. Each bar represents the mean \pm s.d. The results were reproduced in three independent experiments. The asterisk $\left.{ }^{\star}\right)$ indicates $P<0.05$. A full colour version of this figure is available at the British Journal of Cancer journal online.

localisation of MYH9 and ACTN4, and their partial co-localisation with LIMK1 in SW480/HA-LIMK1 cells (Figure 6C).

\section{DISCUSSION}

The LIM kinase family consists of two members; LIMK1 and LIMK2, which play central roles in the rearrangement of the actin cytoskeleton (Scott and Olson, 2007). Numerous research has revealed that LIMK1 may be one of the key molecules that stimulates tumour cell invasion and metastasis, or possibly a new 'oncogene' (Scott and Olson, 2007). So far, the expression and function of LIMK1 in CRC development and progression is still unknown. In the present study, we investigated for the first time, the expression of LIMK1 in a large clinical cohort with 152 CRC cases. Our data verified the overexpression of LIMK1 in CRC tissues and its relationship with CRC progression, supporting that LIMK1 might be a novel oncoprotein in CRC.
Our data provided strong evidence that LIMK1 proteins existed not only in the cytoplasm but also in the nucleus of CRC cells. Analysis of the amino-acid sequence of the protein identified that the PDZ of LIMK1 affects nuclear/cytoplasmic shuttling with two leucine-rich NES (Yang et al, 1998; Yang and Mizuno, 1999). Translocation into the nucleus is driven by NLS, which are found between the PDZ and kinase domains in LIMK1. The presence of both NES and NLS suggests that nuclear/cytoplasmic shuttling might be a regulated process and that LIMK1 may have active functions in the nucleus (Goyal et al, 2006). Our results indicated that both nuclear and cytoplasmic-localised HA-LIMK1 promoted xenograft tumour growth and lung homing capacity in nude mice. A significant correlation was found between LIMK1 overexpression of distinct compartments and worse clinical prognosis, probably resulting from the LIMK1-mediated aggressive phenotype of CRC cells.

Despite the clinical and biological significance of LIMK1 in tumour progression, the downstream mechanism and molecular 

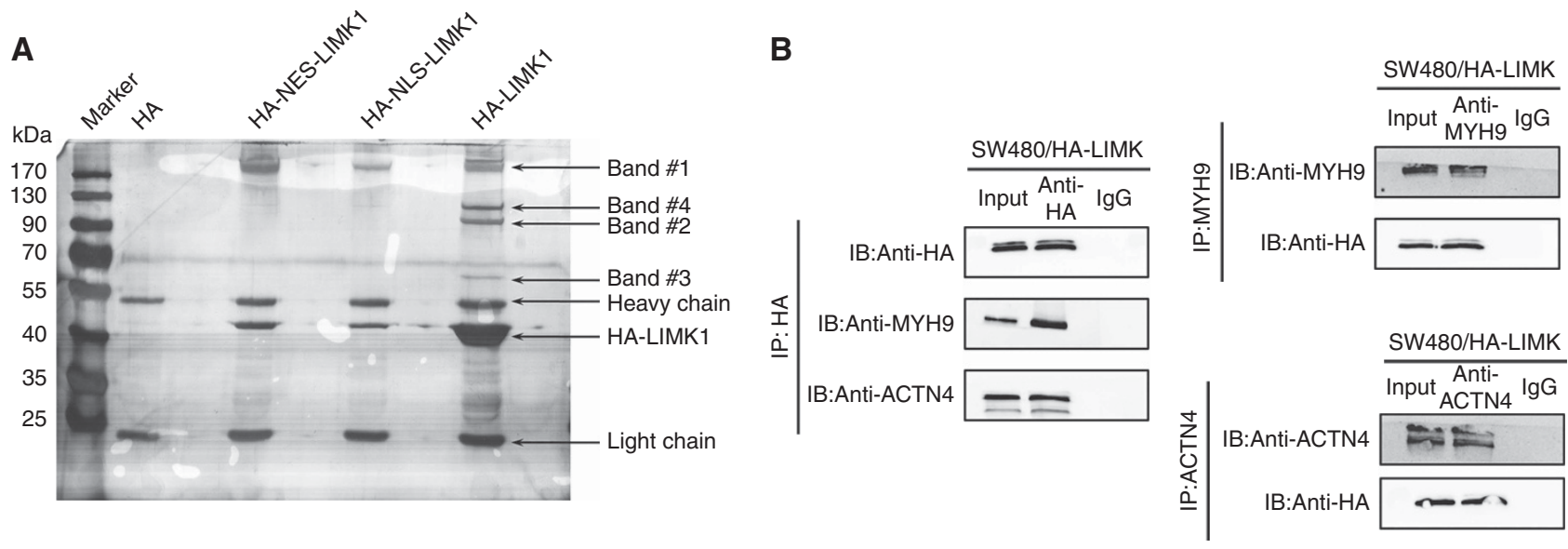

\section{C} SW480/HA-LIMK1
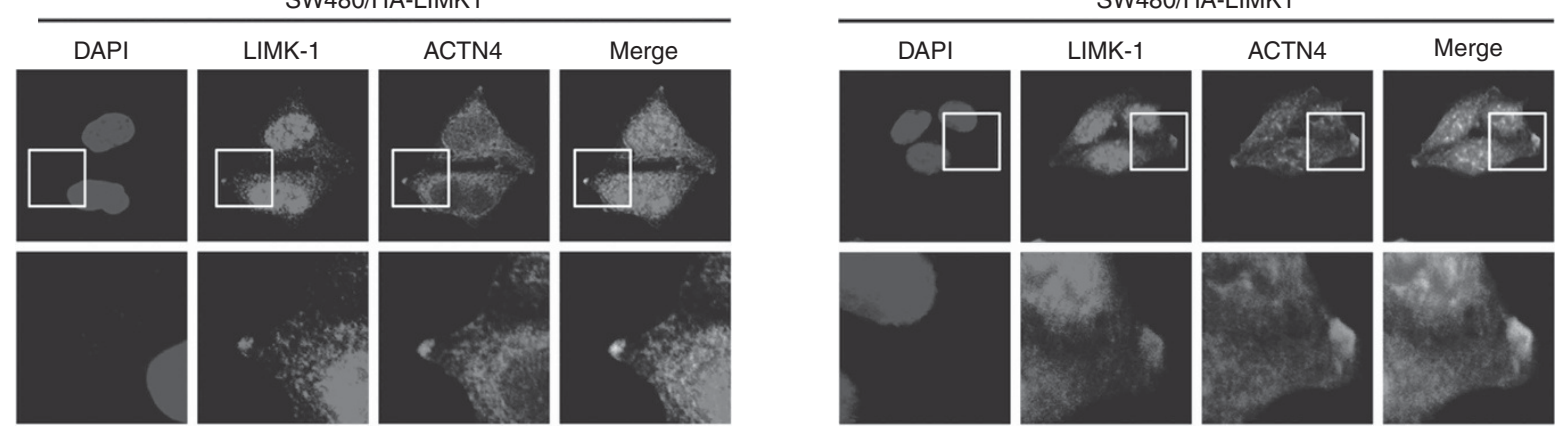

Figure 6. MYH9 and ACTN4 are downstream factors of LIMK1. (A) Immunoprecipitation of the whole proteins from SW480/HA-LIMK1, SW480/ HA-NES-LIMK1 and SW480/HA-NLS-LIMK1 cells with anti-HA antibody, respectively. (B) Validation of endogenous interaction between LIMK1 and MYH9 or ACTN4 in indicated cells. The input sample contained $2 \%$ of the total proteins used for the immunoprecipitation. (C) The subcellular localisation of MYH9 or ACTN4 and its co-localisation with LIMK1 in indicated cells was assessed by confocal microscopy (original magnification $\times 2400$ ). A full colour version of this figure is available at the British Journal of Cancer journal online.

targets of LIMK1 remains to be elucidated. Not surprisingly, studies on LIMK1 have until now focused on the role of LIMK1 in the actin cytoskeleton (Scott and Olson, 2007). An integrin-linked machinery of cytoskeletal regulation involving LIMK1 contributed to tumour initiation and metastatic colonisation by inducing EMT (Shibue et al, 2013). We further explored the proteins interacting with LIMK1 in CRC cells via a protein-protein interaction technique. Among the candidate proteins, MYH9 and ACTN4 were identified as two novel targets of LIMK1. Knockdown of MYH9 or ACTN4 partly counteracted the malignant phenotypes mediated by LIMK1, suggesting their essential role in CRC progression.

MYH9, a gene encoding nonmuscle myosin IIA (NMIIA), belongs to the myosin II subfamily of actin-based molecular motors that includes skeletal, cardiac, smooth muscle, and nonmuscle myosins (Simons et al, 1991; Toothaker et al, 1991). Several studies have indicated that MYH9 plays a key role in cancer cell invasion and metastasis (Betapudi et al, 2006; Derycke et al, 2011). However, a study based on a direct in vivo RNAi strategy showed that MYH9 RNAi and MYH9 knockout trigger invasive squamous cell carcinoma (SCC) formation on tumour-susceptible backgrounds. MYH9 is diminished in human SCCs with poor survival, suggesting it as a potential tumour suppressor (Schramek et al, 2014). Although further studies need to be carried out, our data provide new evidence that MYH9 might serve as a novel CRC metastasis-associated protein.

ACTN4, a member of the cytoskeletal protein family, binds to actin filaments to preserve cytoskeletal structure and cell morphology (Condeelis and Segall, 2003; Ebashi and Ebashi, 1964; Goto et al, 2003). ACTN4 is expressed in nonmuscle cells and has been frequently reported to be associated with cell motility and cancer metastasis (Honda et al, 1998). During cell movement, ACTN4 protein expression level is markedly increased and ACTN4 concentrates at the leading edge of migrating cells (Honda et al, 1998). ACTN4 knockdown suppresses the migration and invasion of cancer cells (Kikuchi et al, 2008; Yoshii et al, 2013; Shao et al, 2014), whereas its overexpression in CRC cells induces lymph node metastasis in immunodeficient mice (Honda et al, 2005). Our data showed that ACTN4 knockdown suppressed actin cytoskeleton and cell migration of LIMK1-overexpressing cells, supporting its essential role in LIMK1-mediated CRC progression.

In summary, we observed that LIMK1 was upregulated in CRC, especially in those with metastatic potential, implying its relationship with poor clinical outcome. Notably, both cytoplasmic and nuclear LIMK1 overexpression functionally promoted CRC cell aggressiveness both in vitro and in vivo by driving EMT and activating the PI3K/Akt signalling pathway. Moreover, two tumour metastasis-associated proteins, MYH9 and ACTN4, were identified as direct targets of LIMK1 and are required for LIMK1-mediated aggressive phenotype. Therefore, LIMK1 provides clues for novel diagnostic strategies and specific targeted drugs for managing advanced CRC patients.

\section{ACKNOWLEDGEMENTS}

This work was supported by the National Natural Science Foundation of China (Nos. 81572813), Guangdong Natural Science Funds for Distinguished Young Scholar (S20120011334), Guangdong Natural Science Foundation (2014A030313490, 
2015A030313274), and Science and Technology Program of Guangzhou (1563000235).

\section{CONFLICT OF INTEREST}

The authors declare no conflict of interest.

\section{AUTHOR CONTRIBUTIONS}

LZ led study design and prepared the manuscript. QL, RL, and ZP carried out the experiments; RZ performed statistical analysis. LX assisted in tissue sample collection and clinical analysis. YD performed data analysis and interpretation.

\section{REFERENCES}

Bagheri-Yarmand R, Mazumdar A, Sahin AA, Kumar R (2006) LIM kinase 1 increases tumor metastasis of human breast cancer cells via regulation of the urokinase-type plasminogen activator system. Int J Cancer 118(11): 2703-2710.

Betapudi V, Licate LS, Egelhoff TT (2006) Distinct roles of nonmuscle myosin II isoforms in the regulation of MDA-MB-231 breast cancer cell spreading and migration. Cancer Res 66(9): 4725-4733.

Chen P, Zeng M, Zhao Y, Fang X (2014) Upregulation of Limk1 caused by microRNA-138 loss aggravates the metastasis of ovarian cancer by activation of Limk1/cofilin signaling. Oncol Rep 32(5): 2070-2076.

Condeelis J, Segall JE (2003) Intravital imaging of cell movement in tumours. Nat Rev Cancer 3(12): 921-930.

Davila M, Frost AR, Grizzle WE, Chakrabarti R (2003) LIM kinase 1 is essential for the invasive growth of prostate epithelial cells: implications in prostate cancer. J Biol Chem 278(38): 36868-36875.

Derycke L, Stove C, Vercoutter-Edouart AS, De Wever O, Dolle L, Colpaert N, Depypere H, Michalski JC, Bracke M (2011) The role of non-muscle myosin IIA in aggregation and invasion of human MCF-7 breast cancer cells. Int J Dev Biol 55(7-9): 835-840.

Ebashi S, Ebashi F (1964) A new protein factor promoting contraction of actomyosin. Nature 203: 645-646.

Goto H, Wakui H, Komatsuda A, Ohtani H, Imai H, Sawada K, Kobayashi R (2003) Renal alpha-actinin-4: purification and puromycin aminonucleoside-binding property. Nephron Exp Nephrol 93(1): e27-e35.

Goyal P, Pandey D, Siess W (2006) Phosphorylation-dependent regulation of unique nuclear and nucleolar localization signals of LIM kinase 2 in endothelial cells. J Biol Chem 281(35): 25223-25230.

Gupta GP, Massague J (2006) Cancer metastasis: building a framework. Cell 127(4): 679-695.

Honda K, Yamada T, Endo R, Ino Y, Gotoh M, Tsuda H, Yamada Y, Chiba H, Hirohashi S (1998) Actinin-4, a novel actin-bundling protein associated with cell motility and cancer invasion. J Cell Biol 140(6): 1383-1393.

Honda K, Yamada T, Hayashida Y, Idogawa M, Sato S, Hasegawa F, Ino Y, Ono M, Hirohashi S (2005) Actinin- 4 increases cell motility and promotes lymph node metastasis of colorectal cancer. Gastroenterology 128(1): 51-62.

Kikuchi S, Honda K, Tsuda H, Hiraoka N, Imoto I, Kosuge T, Umaki T, Onozato K, Shitashige M, Yamaguchi U, Ono M, Tsuchida A, Aoki T, Inazawa J, Hirohashi S, Yamada T (2008) Expression and gene amplification of actinin- 4 in invasive ductal carcinoma of the pancreas. Clin Cancer Res 14(17): 5348-5356.

Li R, Doherty J, Antonipillai J, Chen S, Devlin M, Visser K, Baell J, Street I, Anderson RL, Bernard O (2013) LIM kinase inhibition reduces breast cancer growth and invasiveness but systemic inhibition does not reduce metastasis in mice. Clin Exp Metastasis 30(4): 483-495.

Mizuno K, Okano I, Ohashi K, Nunoue K, Kuma K, Miyata T, Nakamura T (1994) Identification of a human cDNA encoding a novel protein kinase with two repeats of the LIM/double zinc finger motif. Oncogene 9(6): $1605-1612$.
Nunoue K, Ohashi K, Okano I, Mizuno K (1995) LIMK-1 and LIMK-2, two members of a LIM motif-containing protein kinase family. Oncogene 11(4): 701-710.

Ohashi K, Hosoya T, Takahashi K, Hing H, Mizuno K (2000) A Drosophila homolog of LIM-kinase phosphorylates cofilin and induces actin cytoskeletal reorganization. Biochem Biophys Res Commun 276(3): 1178-1185.

Okano I, Hiraoka J, Otera H, Nunoue K, Ohashi K, Iwashita S, Hirai M, Mizuno K (1995) Identification and characterization of a novel family of serine/threonine kinases containing two N-terminal LIM motifs. J Biol Chem 270(52): 31321-31330.

Prunier C, Josserand V, Vollaire J, Beerling E, Petropoulos C, Destaing O, Montemagno C, Hurbin A, Prudent R, de Koning L, Kapur R, Cohen PA, Albiges-Rizo C, Coll JL, van Rheenen J, Billaud M, Lafanechere L (2016) LIM kinase inhibitor Pyr1 reduces the growth and metastatic load of breast cancers. Cancer Res 76(12): 3541-3552.

Schramek D, Sendoel A, Segal JP, Beronja S, Heller E, Oristian D, Reva B, Fuchs E (2014) Direct in vivo RNAi screen unveils myosin IIa as a tumor suppressor of squamous cell carcinomas. Science 343(6168): 309-313.

Scott RW, Olson MF (2007) LIM kinases: function, regulation and association with human disease. J Mol Med 85(6): 555-568.

Shao H, Li S, Watkins SC, Wells A (2014) alpha-Actinin-4 is required for amoeboid-type invasiveness of melanoma cells. J Biol Chem 289(47): 32717-32728.

Shibue T, Brooks MW, Weinberg RA (2013) An integrin-linked machinery of cytoskeletal regulation that enables experimental tumor initiation and metastatic colonization. Cancer Cell 24(4): 481-498.

Siegel R, Desantis C, Jemal A (2014) Colorectal cancer statistics, 2014. CA Cancer J Clin 64(2): 104-117.

Simons M, Wang M, McBride OW, Kawamoto S, Yamakawa K, Gdula D, Adelstein RS, Weir L (1991) Human nonmuscle myosin heavy chains are encoded by two genes located on different chromosomes. Circ Res 69(2): 530-539.

Spano D, Heck C, De Antonellis P, Christofori G, Zollo M (2012) Molecular networks that regulate cancer metastasis. Semin Cancer Biol 22(3): 234-249.

Toothaker LE, Gonzalez DA, Tung N, Lemons RS, Le Beau MM, Arnaout MA, Clayton LK, Tenen DG (1991) Cellular myosin heavy chain in human leukocytes: isolation of 5' cDNA clones, characterization of the protein, chromosomal localization, and upregulation during myeloid differentiation. Blood 78(7): 1826-1833.

Wan L, Zhang L, Fan K, Wang J (2014) MiR-27b targets LIMK1 to inhibit growth and invasion of NSCLC cells. Mol Cell Biochem 390(1-2): $85-91$.

Yang N, Higuchi O, Mizuno K (1998) Cytoplasmic localization of LIM-kinase 1 is directed by a short sequence within the PDZ domain. Exp Cell Res 241(1): 242-252.

Yang N, Mizuno K (1999) Nuclear export of LIM-kinase 1, mediated by two leucine-rich nuclear-export signals within the PDZ domain. Biochem J 338(Pt 3): 793-798.

Yoshii H, Ito K, Asano T, Horiguchi A, Hayakawa M (2013) Increased expression of alpha-actinin- 4 is associated with unfavorable pathological features and invasiveness of bladder cancer. Oncol Rep 30(3): 1073-1080.

You T, Gao W, Wei J, Jin X, Zhao Z, Wang C, Li Y (2015) Overexpression of LIMK1 promotes tumor growth and metastasis in gastric cancer. Biomed Pharmacother 69: 96-101.

Zhang YF, Liu L, Ding YQ (2007) Isolation and characterization of human colorectal cancer cell subline with unique metastatic potential in the liver. Nan Fang Yi Ke Da Xue Xue Bao 27(2): 126-130.

Zhao L, Wang H, Liu C, Liu Y, Wang X, Wang S, Sun X, Li J, Deng Y, Jiang Y, Ding Y (2010) Promotion of colorectal cancer growth and metastasis by the LIM and SH3 domain protein 1. Gut 59(9): 1226-1235.

This work is published under the standard license to publish agreement. After 12 months the work will become freely available and the license terms will switch to a Creative Commons AttributionNonCommercial-Share Alike 4.0 Unported License.

Supplementary Information accompanies this paper on British Journal of Cancer website (http://www.nature.com/bjc) 